\title{
Interferon Beta-1b for the Treatment of Multiple Sclerosis - More than 10 Years of Experience
}

\author{
Laura lulia Barcutean ${ }^{1,2}$, Smaranda Maier 1,2 , Zoltan Bajko1,2, Anca Motataianu'1,2, Andreea Romaniuc², \\ Sebastian Razvan Andone ${ }^{2^{*}}$, Rodica loana Balasa ${ }^{1,2}$ \\ 1. University of Medicine, Pharmacy, Sciences and Technology of Târgu Mureș, Romania, \\ 2. Mures County Emergency Clinical Hospital, Department of Neurology, Târgu Mureș, Romania
}

\begin{abstract}
Objective: Interferon beta-1b (IFN $\beta-1 b)$ was the first disease-modifying agent (DMT) used for the treatment of multiple sclerosis (MS). We aimed to evaluate the first patients with MS that started treatment in our clinic. Methods: An observational, retrospective study was performed on 78 patients that had continuous treatment with IFN $\beta-1 b$ for more than 10 years. The collection of the demographical data and periodical clinical evaluation was performed on all patients. The disability was quantified using the Expanded Disability Status Scale (EDSS), creating two groups of patients, G1: EDSS < 4.0 and G2: EDSS $\geq 4.0$. The hallmarks of the disability evolution were gathered by direct patient interview, such as the symptoms at onset and relapse frequency. Results: After more than 17 years of disease evolution, more than half (65.38\%) of the patients present a mild disability score. The majority (54.90\%) started treatment in the first three years after the onset, while the patients in G2 started treatment after more than 3 years from the onset. The initiation of IFN $\beta-1 \mathrm{~b}$ lead to a significant reduction of the relapse rates. A reduced number of patients (<25\%) transitioned from RRMS to SPMS. Discussion: Continuous evaluation of MS patients allows us to assess the possibility of prolonged treatment with IFN $\beta-1 \mathrm{~b}$ and to differentiate the responders from non-responders. The clear reduction in relapse rates and disability progression, notably in patients that started treatment early ensure us into continuing administering this medication. Compared to historical cohorts, our lot had a slower disability evolution and a significant proportion hadn't reach an important disability score.
\end{abstract}

Keywords: multiple sclerosis, interferon $\beta-1 b$, disability, evolution, disease-modifying therapy

Received 14 November 2018 / Accepted 5 March 2019

\section{Introduction}

Multiple sclerosis (MS), a chronic, immune-mediated disease of the central nervous system (CNS), which predominantly affects the young adults, is one of the most frequent causes of neurological disability in the modern world. Relapsing-remitting MS (RRMS) is the most common form of evolution, which accounts for most of the cases and usually converts into the secondary-progressive MS (SPMS) in 10 to 15 years. $[1,2]$ Accumulation of disability in MS is secondary to both inflammatory and neurodegenerative processes. From a clinical perspective, active inflammation will trigger a relapse and recovery may be complete or partial, resulting in neurological sequelae. Neurodegenerative processes will lead to progressive disability and mark the onset of the SPMS [3]. The available treatments for MS exert their actions mainly in the inflammatory phase by modulating the immune response, thus being known as disease-modifying therapies (DMT).

The interferons were discovered by accident in 1957 by Alick Isaacs and Jean Lindenmann, two British researchers who were actively following the viral response to the Influenza viruses in animal models (chicken embryos) [4]. While the interferon gamma was the first agent used for the treatment of MS, it proved to have a contrasting effect by incrementing the relapse rates and overall augmenting the disease evolution [5], being quickly replaced by the in-

* Correspondence to: Sebastian Razvan Andone

E-mail: adn.sebastian.007@gmail.com terferon beta. One of the most studied DMTs - the interferon beta was the first immunomodulatory agent used for the treatment of MS, with a well-known safety profile and a good impact upon the general clinical evolution of the patients. The interferon beta-1b (IFN $\beta-1 b)$ was approved for the treatment of RRMS in USA in 1993, and in EU in 1995 [6], and shortly after, in 1999, it was approved for use in SPMS [7] after extensive studies that focused upon disease's evolution determined that it also reduces the disability progression and [8] controls the relapse rates in patients with an SPMS $[9,10]$.

Despite the increasing number of DMTs appearing on the market, the IFN $\beta-1 \mathrm{~b}$ remains one of the most prescribed agents for MS' treatment. Whether this drug can significantly delay the disability accumulation when being used for long periods of time is still under dispute, but due to its worldwide availability, the increasing number of long-term treated cohorts of patients starts to answer one question at the time. In order to bring our contribution to the MS world, we examined the clinical impact of IFN $\beta$ $1 \mathrm{~b}$ in MS patients treated continuously for more than 10 years.

\section{Methods \\ Lot selection}

For this study, patients treated with IFN $\beta-1 b$ subcutaneously once every two days were selected. In our clinic we treat more than 400 patients with MS, from which 140 are treated with IFN $\beta-1 b$. 
An observational, retrospective study was performed on 78 patients that had continuous, uninterrupted treatment with IFN $\beta-1 \mathrm{~b}$ for more than a decade. Data collection and patient evaluation took place between 2016-2017. The patients have been followed ever since the treatment was instituted in the Neurology 1 Clinic of the Emergency Clinical County Hospital of Targu Mures, Romania. All the patients that are included in the MS treatment programme were evaluated every six months or when needed in case of relapses or other symptoms, as well as brain and spinal cord magnetic resonance imagining (MRI) together with complete neurological follow-ups, as per diagnosis protocol based on the revised McDonalds criteria (2010). [11]

The inclusion criteria were: a) patients diagnosed with MS based on McDonalds criteria revised in 2010, b) were treated continuously for more than 10 years with IFN $\beta$ $1 \mathrm{~b}, \mathrm{c}$ ) had no prior immunomodulatory treatment and d) consented to regular follow-ups and to be a part of the study. The exclusion criteria were applied for the remaining 62 patients, as following: a) patients interrupted at one point, either voluntarily (pregnancy) or because of adverse effects the treatment with IFN $\beta-1 b$, b) had prior immunomodulatory treatment with other DMT, c) they did not consent to be a part of the study, d) had less than 10 years of continuous treatment with IFN $\beta-1 b$.

\section{Lot evaluation}

The patient's demographical and clinical data, including date of the clinical onset, symptoms at onset, relapses and disability were recorded at the first visit and historical information about the course of the disease was retrospectively collected and analysed. The neurological disability was quantified using the Expanded Disability Status Scale (EDSS) [12], actively following the disability progression and moment of conversion from a RR to a SP. The functional systems (FS) affected at onset were noted as optic (O-FS): onset with optic neuritis, pyramidal (P-FS): onset with pure motor deficit, brainstem (B-FS): onset with diplopia or alternate syndromes, cerebellar (C-FS): onset with coordination impairment and instability, sensory (SFS): onset with either paraesthesias or loss of sensibility and polysymptomatic onset(X-FS).

The total number of relapses was calculated as annual relapse ratio (ARR), by using the following formula: ARR = number of relapses/number of treatment years. ARR was defined as the total number of relapses, ARR_0 as relapses before the treatment respectively, ARR_1, relapses on treatment. A relapse was considered as the sudden onset of new neurological symptoms with a concomitant worsening of the clinical picture, that occurred in the absence of fever or any other kind of active infection. The events lasted more than 24 hours and alleviated spontaneously or with the use of corticosteroids [13].

The disability score was noted according to EDSS, and to simplify the patient selection two groups were defined: G1 - patients with an EDSS $<4.0$ and G2 - patients with an EDSS $\geq 4.0$ at the study inclusion. The patients' evolutions were followed accordingly, thus the progression was subsequently assessed by consulting the old records, mainly the transition from a lesser EDSS to a score of 4.0 or 6.0. The milestones 4.0 and 6.0 were chose because of the impact they have upon the disability progression. Thus, an irreversible EDSS of 4.0 marks the first clinical signs of ambulatory restriction, limiting the walking perimeter within 500 meters (without any physical aid), and the EDSS of 6.0 is defined by the necessity of unilateral assistance for ambulation. By evaluating the patient files, we were able to indicate, when applicable, the moment of progression, keeping in mind that the ambulatory capacity is the main determinant for the EDSS. The EDSS at the start of the treatment is EDSS_0, respectively at the study inclusion EDSS_1.

\section{Statistical Analysis}

The data was centralized using the Excel Platform incorporated in Microsoft Office 2016 and the statistical analysis was performed using Graph Pad Prism 6. We used mean and standard deviation (SD) when assessing gaussian population, and median and range defined by $25 \%-75 \%$ percentile when analysing non-gaussian populations. The normality tests used were Shapiro-Wilk and KolmogorovSmirnov, and the correlations were performed using Spearman or Pearson test, after assessing the normality distribution. The statistical significance was defined when $\mathrm{p}<0.05$.

\section{Results}

A total of 78 MS patients that had continuous treatment with IFN $\beta-1 b$ for more than 10 years were evaluated. Patient demographic and general clinical data are presented in Table I. At the moment of study inclusion, 51 (65.38\%) of the patients had an EDSS lower than 4.0, while 27 (34.61\%) had a disability score higher or equal to 4.0 . The female gender is dominant in both groups, $68.62 \%$ in G1 and $70.37 \%$ in G2. There was no statistically significant difference $(p=0.259)$ when comparing the age at onset of the disease for both groups, (Fig. 1) but statistical significant data was found when comparing the age at the beginning of the treatment between the two groups $(\mathrm{p}<0.0001)$, with a median for $\mathrm{G} 1$ of 35 years, respectively 42 years for G2 (Fig 1). Most of the patients in G1 (54.90\%) started treatment between 1 and 3 years from the onset of the symptoms, while in G2, most of them started treatment after 3 years $(70.22 \%)$.

From the cohort of patients, $63(80.75 \%)$ started treatment with an EDSS between 0 and 3.5 and 48 (76.16\%) of them still have an EDSS $<4.0$ at the moment of study inclusion, signifying that more than three quarters of the patients with a mild/moderate degree of disability still have a good clinical course. $15(23.80 \%)$ patients converted to SPMS.

The patients had been treated with IFN $\beta-1 b$ approximately 13 years for both groups. The mean duration of 
Table I. Demographic and clinical data of the patients

\begin{tabular}{|c|c|c|c|c|}
\hline & $\begin{array}{c}\text { All patients - } \mathrm{G} \\
\mathrm{n}=78\end{array}$ & $\begin{array}{c}\text { EDSS }<4.0-\mathrm{G1} \\
\mathrm{n}=51(65.38 \%)\end{array}$ & $\begin{array}{c}\text { EDSS } \geq 4.0-\text { G2 } \\
n=27(34.61 \%)\end{array}$ & $\begin{array}{c}\text { EDSS } \leq 2.0 \\
n=29(38.46 \%)\end{array}$ \\
\hline Female & $54(69.23 \%)$ & $35(68.62 \%)$ & $19(70.37 \%)$ & $23(79.31 \%)$ \\
\hline Male & $24(30.76 \%)$ & $16(31.37 \%)$ & $8(29.62 \%)$ & $6(20.68 \%)$ \\
\hline$<20$ years old & $4(5.12 \%)$ & $3(5.88 \%)$ & $1(3.70 \%)$ & $1(3.44 \%)$ \\
\hline 20-30 years old & $35(44.87 \%)$ & $21(41.17 \%)$ & $14(51.85 \%)$ & $13(44.82 \%)$ \\
\hline $31-40$ years old & $30(38.46 \%)$ & $26(50.98 \%)$ & $4(14.81 \%)$ & $12(41.37 \%)$ \\
\hline Mean age at the beginning of treatment $\left( \pm \mathrm{SD}^{\star}\right)$ (years) & $36.73 \pm 8.53$ & $29.82 \pm 7.19$ & $42.04 \pm 7.52$ & $29.72 \pm 7.26$ \\
\hline Mean duration of the disease (years) $( \pm S D)$ & $18.90 \pm 7.10$ & $16.90 \pm 5.43$ & $22.67 \pm 8.36$ & $16.10 \pm 5.010$ \\
\hline Mean treatment duration (years) $( \pm S D)$ & $13.08 \pm 2.46$ & $12.80 \pm 2.38$ & $13.59 \pm 2.59$ & $12.34 \pm 2.05$ \\
\hline \multicolumn{5}{|l|}{ Start treatment } \\
\hline Same year & $9(11.53 \%)$ & $7(13.72 \%)$ & $2(7.40 \%)$ & $2(6.89 \%)$ \\
\hline $1-3$ years & $34(43.58 \%)$ & $28(54.90 \%)$ & $6(22.22 \%)$ & $18(62.06 \%)$ \\
\hline$>3$ years & $35(44.87 \%)$ & $16(31.37 \%)$ & $19(70.22 \%)$ & $9(31.03 \%)$ \\
\hline Optic (O-FS) & $16(20.51 \%)$ & $13(25.49 \%)$ & $3(11.11 \%)$ & $9(31.03 \%)$ \\
\hline Pyramidal (P-FS) & $19(24.35 \%)$ & $12(23.52 \%)$ & $7(25.92 \%)$ & $5(17.24 \%)$ \\
\hline Cerebellar (C-FS) & $6(7.69 \%)$ & $2(3.92 \%)$ & $4(14.81 \%)$ & $1(3.44 \%)$ \\
\hline Sensory (S-FS) & $11(14.10 \%)$ & $8(15.68 \%)$ & $3(11.11 \%)$ & $5(17.24 \%)$ \\
\hline Brainstem (B-FS) & $20(25.64 \%)$ & $13(25.49 \%)$ & $7(25.92 \%)$ & $6(20.68 \%)$ \\
\hline Polysymptomatic (X-FS) & $6(7.69 \%)$ & $3(5.88 \%)$ & $3(11.11 \%)$ & $3(10.34 \%)$ \\
\hline Median EDSS_0 (25\%-75\% percentile) & $2.0(1.0-3.0)$ & $1.5(1.0-2.5)$ & $3.5(2.5-4.5)$ & $1.5(1.0-2.0)$ \\
\hline Median EDSS_1 (25\% - 75\% percentile) & $3.5(2.0-5.5)$ & $2.0(1.5-3.5)$ & $6.0(5.0-6.5)$ & $1.5(1.0-2.0)$ \\
\hline
\end{tabular}

*SD: standard deviation

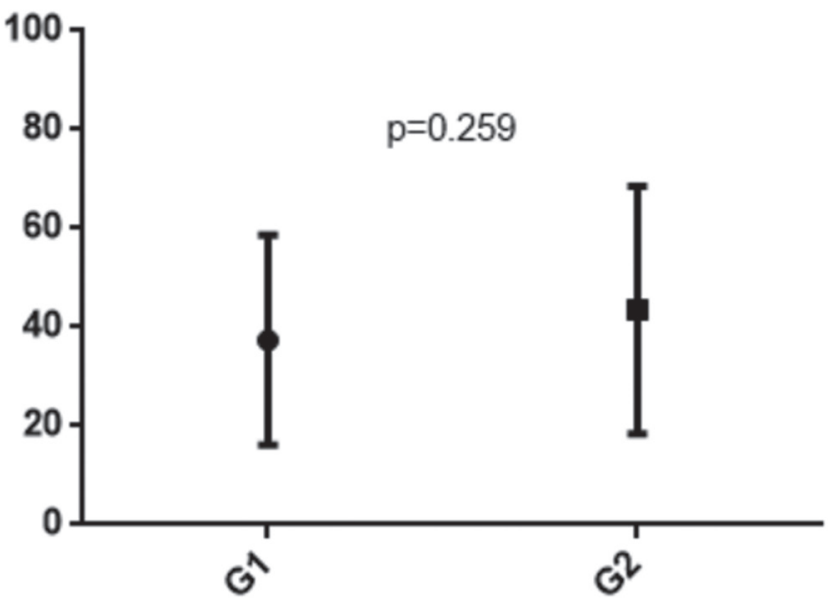

Age at onset of the disease for G1 and G2 (Mann Whitney test)

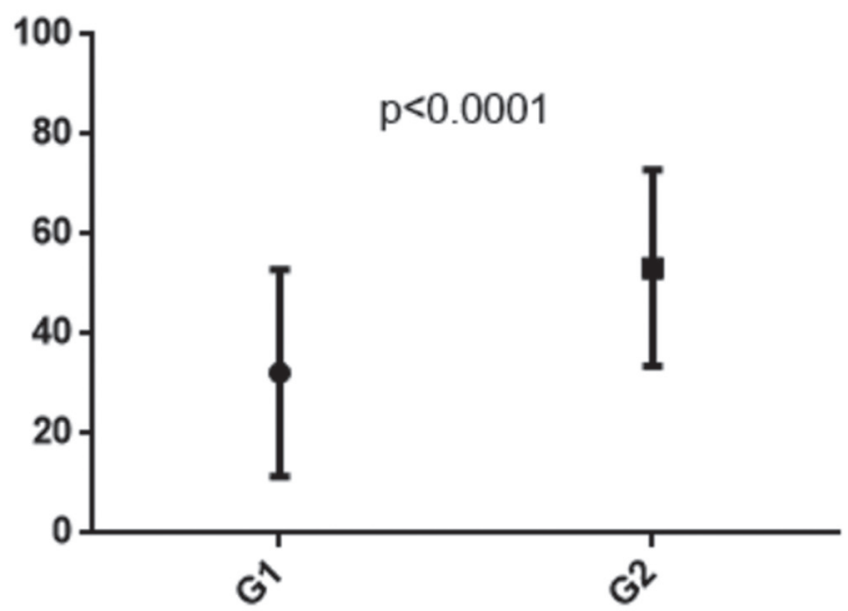

Age at beginning of the treatment for G1 and G2 (Mann Whitney test)

Fig. 1. Age values analysis for G1 and G2, based on the onset and moment of treatment initiation

the disease was 16.90 years for G1 and 22.67 years for G2. Most of our patients in G1 presented at onset with B-FS and O-FS (25.49\%) symptoms, while in G2, the predominance was between B-FS and P-FS (25.92\%). The EDSS_0 ranged from 1.5 in G1 to 3.5 in G2. At the study inclusion, the EDSS_1 for G1 was 2.0, while for G2 was 6.0.

By comparing the ARR before and after the treatment was instituted, statistical significance was found for all the groups: G, G1, and G2, but with a stronger p-value for G1 $(p<0.0001)$, signifying a reduced relapse ratio after treatment with IFN $\beta-1 b$ was started (Fig 2).

The overview of the clinical evolution of the patients is presented in Table II. This included the 15 patients that converted to an SPMS during the treatment. We did not account for patients that already had a higher disability level when they started treatment, because of the difficulty in correctly assessing the moment of conversion historically. The median time from the MS' onset to an EDSS of 4.0 was 12 years and for an EDSS of 6.0 was 16 years. The mean treatment duration was $15 \pm 2.63$ years. This can be interpreted as following in our study: a patient will reach

Table II: Conversion of the RRMS patients into SPMS

\begin{tabular}{lcc}
\hline $\mathbf{n}=\mathbf{1 5}$ & EDSS 4.0 & EDSS 6.0 \\
\hline $\begin{array}{l}\text { MS' onset (years) } \\
\text { Median (25\% }-75 \% \text { percentile) }\end{array}$ & $12(8-17)$ & $16(9-22)$ \\
$\begin{array}{l}\text { Start of treatment (years) } \\
\text { Median (25\% - 75\% percentile) }\end{array}$ & $5(1-7)$ & $6(1.5-10)$
\end{tabular}




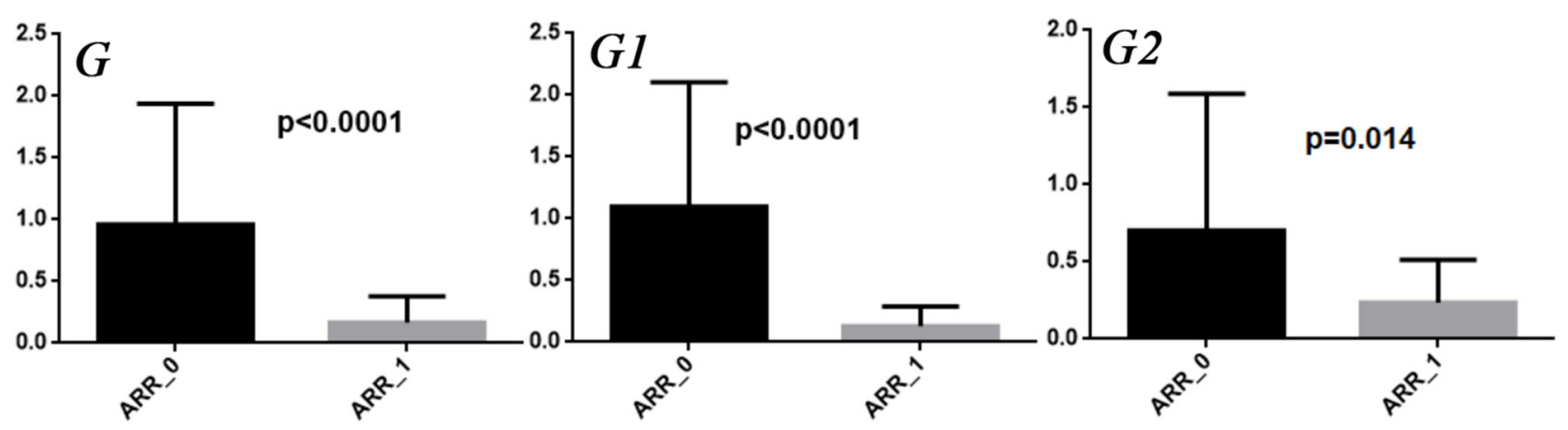

Fig. 2. Analysis of the relapse rates using Wilcoxon matched paired signed ranked test.

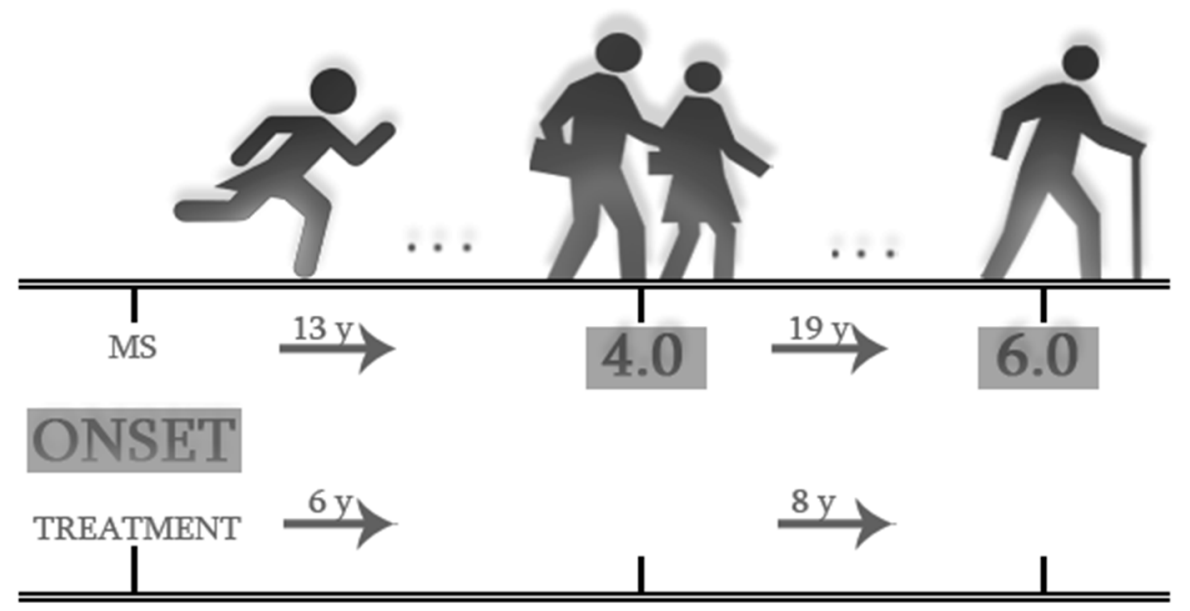

Fig. 3. Representation of the evolution of disability

an irreversible EDSS of 6.0 in 4 years after the start of the progressive phase. The median time from the start of the treatment to an EDSS of 4.0 was 5 years and to an EDSS of 6.0 was 6 years. The evolution towards an SP phase is represented in Fig.3.

The patients were evaluated every 6 months for the entire duration of the treatment. The retrospective information allowed us also to explore the subgroup with the lesser degree of disability and to assess the clinical data (Table I). 29 (38.46\%) patients present with an EDSS $<2.0$ at the moment of study inclusion. Their mean age at MS' onset, the mean age at the beginning of the treatment, mean duration of the disease and mean treatment duration were similar between the G1 and G2. Most of the patients started IFN $\beta-1 b$ in the first three years from the onset $(62.06 \%)$ and the dominant FS present at onset was O-FS (31.03\%), followed by B-FS (20.68\%). The median EDSS_0 was equal to EDSS_1, with a low disability score of 1.5 .

\section{Discussion}

The efficiency of IFN $\beta-1 b$ has been the subject of numerous individual and collective studies, mainly due to its availability and ease of monitoring. Ever since its approval in 1993, numerous extensions followed, which demonstrated that continuous administration has a persistent effect upon relapse rate reduction [14], thus diminishing the disability accumulation. In our present study, we focused on clinically evaluating those patients that started the treatment as soon as it became available in our country, know- ing that IFN $\beta-1 \mathrm{~b}$ was first DMT approved in Romania in our center in 2000 for the RRMS and, shortly after, for the SPMS.

The pathophysiological mechanisms of MS, though not being fully understood yet, are characterized by two entwining processes: demyelination and neurodegeneration, defining MS as a two-stage disease [15]. Demyelination is the result of an over-active auto-immunity and is characteristic for the active form of the disease, RRMS, where inflammation is the dominating pathophysiological process and usually manifests as clinical relapses. The available DMTs will exert their action modulating the inflammation and have a modest effect on the second stage, the neurodegeneration, marked by axonal loss. But the evolution of disability in the first phase seems to be independent than the progression that manifests in the second phase of the disease [16].

IFN $\beta-1 \mathrm{~b}$ mechanism of action is partially known, but it's main effects are targeted against lymphocyte $\mathrm{T}$ proliferation and a reduction of endogenous IFN-gamma. It also blocks the class II major histocompatibility complex (MHC) and reduces the subsequent expression of the antigen presentation, blocking the activity of the adhesion molecules, reducing thus the inflammation in the CNS [17]. At a umoral level, the agents act upon reduction of the pro-inflammatory cytokines and augment the expression of anti-inflammatory agents [18].

The demographic data of our patients were similar to the results reported by other studies. The obvious dominance 
was for female patients [19]. There is a genetic susceptibility for female patients to have a higher risk of developing auto-immune conditions [20], some linked to MHC genes on chromosome 6 [21] and some explained by the various HLA complexes [22]. We found no significant differences between the age of the patients at the MS onset. What was expected was the strong statistical significance when we evaluated the age of patients at the start of the treatment for both groups, knowing that the onset in younger patients tends to be correlated with a better evolution $[23,24]$.

The importance of early treatment was easily stratified in the present study. More than half of the patients from the G1 started treatment in the first 3 years, while for the G2, most of the patients started treatment over 3 years after onset. Considering the times, it was not uncommon, due to the absence of DMTs in our country when most of our patients presented their first symptoms, but it is clear that early treatment equals better evolution, from the perspective of relapse reduction and disability control [10,25]

Probably one of the most important effects that IFN $\beta$ $1 \mathrm{~b}$ exerted was the reduction in the relapse rates, noticed for both groups but with a stronger statistical significance for the G1. Subsequent relapses will favor disability burden and thus, disability progression, therefore, by controlling relapse rates one can delay the conversion to an SP phase. Several studies hold unto the idea that relapses will increment disability progression [26] while others affirm that the data cannot be validated in order to reproduce this argument [27]. But it is certain that being relapse free is wished for in clinical practice.

IFN $\beta-1 b$ proved its efficiency in significantly delaying the conversion from clinically isolated syndrome (CIS) to clinically defined MS [28]. IFN $\beta-1 b$ effects are also beneficial upon patients with an SP form [17] because even though the effects are mainly anti-inflammatory, by adequately modulating the immune response the agents act upon the residual inflammation that is also present in the second phase of the MS, dominated by neurodegeneration. One study reported a reduction in ARR in SP patients up to a $43 \%$ [29].

The conversion rate from RRMS to SPSM was low, less than $25 \%$ from the patients with RRMS progressed to SPMS. Our patients reached a moderate disability, an EDSS of 4.0 in a median of 12 years and 6.0 in 16 years from the MS' onset. Studies upon the evolution of the disease mention a rate of conversion as high as $54 \%$ [3] with a median time of evolution of 19 years. These patients had a mean time of treatment of 13 years, so we would have anticipated a higher conversion rate. Another study comparing disabilities showed that MS patients will normally reach an EDSS of 6.0 after approximately 17 years of evolution [30], and in our case, this happened at 16 years after the first symptom. The natural history of MS states that, in about 11 years from the onset of the disease, the progression is unavoidable [31]. The mean age of patients that converted to SP in early historical studies was around 40 years [32], but in our study, the patients progressed and reached an irreversible EDSS close to 48 years of age, finding consistent to other international studies on cohorts of long term treated patients [33].

Depending on the onset of the disease, for the G1 the clinical picture was dominated by optic and brainstem symptoms, while the G2 patients the predominance was pyramidal and brainstem symptoms. It has been reported that the onset with ocular [34] or sensory symptoms [35] is usually associated with a lesser degree of disability. A polysymptomatic onset is usually an indicator of a poor prognosis [36].

Seeing how in some of the cases we are faced with a slow or steady evolution, we hypothesized about benign MS. Even though the diagnosis of a benign MS can be made only after a long evolution of the disease and many authors still disapprove of the concept, it's important to evaluate this specific lot of patients in order to at least determine the favourable predictors. Razzolini L et al implied that to maintain a benign MS status, the EDSS should be lower than 2.0 and the diseases' evolution should exceed 10-12 years. Also, brain volume as measured in T1 is an important biomarker that can aid in the diagnosis of a truly benign MS [37]. The selected patients from our cohort with a mild disability level had no significantly different demographic data compared to the whole G1/G2 groups, most of them presented at onset O-FS and B-FS symptoms and they started treatment early in the first 3 years from the onset. The only clinical difference was their EDSS level, at study inclusion their mean EDSS was 1.5, signifying only signs of disability, with no clinical impact. While we cannot say that we have obtained new prognostic factors to aid in the definition of benign MS, the follow-up of the abovementioned patients including imaging studies will aid us into completing the missing diagnosis pieces.

Being one of the most used MS treatment, the safety profile of IFN $\beta$ is well known. One study that followed patients treated with IFN $\beta-1 \mathrm{~b}$ for over 16 years [38] showed that adverse effects (AE) tend to diminish with time. The most frequent $\mathrm{AE}$ are local inflammatory reactions such as pain or tenderness around the area of injections and flu-like syndrome, both of which are easily manageable with intermittent anti-inflammatory medications [39]. Comparing the IFN $\beta$ s, IFN $\beta-1 b$ with subcutaneous administration is better tolerated than IFN $\beta$ - 1 a with subcutaneous/intramuscular administration. [40, 41].

The limitations of our study are represented by the classical method of determining the neurological status of our patients, the EDSS. This scale mainly implies the physical disability and mobility and doesn't quantify the whole clinical picture of an MS patient, such as fatigue, depression, anxiety etc, given the fact that disability might not always be perceived by the patient as the inability to walk. The clinical data of our patients from the first moment they entered the clinic is accurate, as trained MS specialists were performing the clinical evaluations, but the be- 
fore clinical status of the patients, most importantly the ones that joined us already in an SP phase is being left to speculation, since we cannot exactly pinpoint the moment of conversion. An important characteristic of the present study is the uninterrupted IFN $\beta-1 b$ treatment in a rather homogenous population which displays a heterogeneous response.

\section{Conclusions}

Long-term treatment with IFN $\beta$ - $1 \mathrm{~b}$ for MS patients remains a safe and optimal option. It's well-known safety profile and ease of monitoring, manageable $\mathrm{AE}$ and good tolerability makes it one of the most widely prescribed agents for the treatment of MS.

By reducing the relapse rates and disability progression, IFN $\beta$ - $1 \mathrm{~b}$ will delay the evolution of the disease, focusing its effects mainly in the inflammatory phase. It is important to start IFN $\beta-1 \mathrm{~b}$ treatment as early as possible, ideally when the diagnosis of MS has been established, this being universally recommended for any other kind of DMT. Extension of the indication for the treatment of not only RRMS but SPMS makes IFN $\beta-1 b$ an excellent first-line treatment.

However, one must always remember that clinical monitoring it's essential and that if our patients show signs of progression or bad tolerance, either a switch or an escalation of therapy is mandatory in order to ensure the best quality of medical care.

\section{Authors'contributrion}

Laura Iulia Barcutean, MD (Conceptualization; Investigation; Methodology; Writing - original draft; Data analysis) Smaranda Maier, MD, PhD (Reviewing) Zoltan Bajko, MD, PhD (Statistical analysis of the data) Anca Motataianu, MD, PhD (Writing - review \& editing) Andreea Romaniuc, MD (Investigation)

Sebastian Razvan Andone, MD (Investigation; Writing review \& editing)

Rodica Ioana Balasa, MD, PhD, Professor (Conceptualization; Investigation; Writing -review \& editing)

\section{Acknowledgments}

This study was supported by the Doctoral School (I.O.S.U.D) of the University of Medicine and Pharmacy of Tirgu Mures.

\section{Conflict of interest}

The authors have no conflict of interests.

\section{References}

1. Scalfari A, Neuhaus A, Degenhardt A et al - The natural history of multiple sclerosis: a geographically based study 10: relapses and long term disability. Brain. 2010;133:1914-29.

2. Ciccarelli O, Thompson A - Multiple sclerosis in 2015: Managing the complexity of multiple sclerosis. Nat Rev Neurol. 2016;12:70-2.

3. Tremlett H, Yinshan Z, Devonshire V - Natural history of secondaryprogressive multiple sclerosis. Mult Scler. 2008;14:314-24.

4. Isaacs A, Lindenmann J - Virus Interference: I. The Interferon. Proc R
Soc Lond B Biol Sci. 1957;147:258-67.

5. Panitch HS, Hirsch RL, Schindler $J$ et Johnson KP - Treatment of multiple sclerosis with gamma interferon: exacerbations associated with the activation of the immune system. Neurology. 1987;37:1097-102.

6. Kappos L, Polman $\mathrm{CH}$, Freedman MS, et al - Treatment with interferon beta-1b delays conversion to clinically definite and McDonald MS in patients with clinically isolated syndromes. Neurology. 2006;67:1242-9.

7. European Study Group on Interferon Beta-1b in Secondary Progressive MS - Placebo-controlled multicentre randomised trial of interferon beta$1 \mathrm{~b}$ in treatment of secondary progressive multiple sclerosis. Lancet. 1998;1491-7.

8. Filippini G, Munari L, Incorvaia B, et al - Interferons in relapsing remitting multiple sclerosis: a systematic review. Lancet. 2003;361:545-52.

9. Kappos L, Polman C, Pozzilli C, et al - Final analysis of the European multicenter trial on IFNbeta-1b in secondary-progressive MS. Neurology. 2001;57:1969-75.

10. Kappos L, Freedman MS, Polman CH, et al - Long-term effect of early treatment with interferon beta- $1 b$ after a first clinical event suggestive of multiple sclerosis: 5 -year active treatment extension of the phase 3 BENFIT trial. Lancet Neurol. 2009;8:987-997.

11. Polman $\mathrm{CH}$, Reingold SC, Banwell $\mathrm{B}$ et al - Diagnostic criteria for multiple sclerosis: 2010 Revisions to the McDonald criteria. Ann Neurol. 2011;69:292-302

12. Kurtzke JF - Rating neurologic impairment in multiple sclerosis: an expanded disability status scale (EDSS). Neurology. 1983;33:1444-52.

13. Confavreux C, Vukusic S, Thibault M et al. - Relapses and progression of disability in multiple sclerosis. The New England Journal of Medicine. 2000; 343:1430-8.

14. IFNB Multiple sclerosis study group and the University of British Columbia. MS/MRI Analysis group - Interferon beta-1b in the treatment of multiple sclerosis: final outcome of the randomized controlled trial. Neurology. 1995; 45:1277-85.

15. Steinman L - Multiple sclerosis: a two stage disease. Nat Immunol. 2001;2:762-4.

16. Leray E, Yaouaq J, Le Page E et al - Evidence for a two-stage disability progression in multiple sclerosis. Brain. 2010.1900-13.

17. Paolicelli D, Direnzo V, Trojano $M$ - Review of interferon beta $1 \mathrm{~b}$ in the treatment of early and relapsing multiple sclerosis, Biologics. 2009;3:369-76.

18. Barcutean LI, Romaniuc A, Maier S et al - Clinical and serological biomarkers of treatment's response in patients treated continuosly with interferon $\beta$-1b for more than a decade. CNS Neurol Disord Drug Targets. 2018. DOI: 10.2174/1871527317666180917095256. E-pub ahead of print.

19. Harbo JH, Gold R, Tintore M - Sex and gender issues in multiple sclerosis. Ther Adv Neurol Disord. 2013; 6:237-48.

20. Dunn SE, Gunde E, Lee H - Sex -based differences in multiple sclerosis (MS): Part II: Rising incidence of multiple sclerosis women and the vulnerability of men to progression of this disease. Current Topics in behavioural neurosciences. 2015;26:57-86.

21. Bell J, Lathrop G - Multiple loci for multiple sclerosis. Nat Genet. 13;1996:377-8.

22. Oksenberg J, Barcellos $L$, Cree $L$ et al - Mapping multiple sclerosis susceptibility to the HLA-DR locus in African americans. Am J Hum Genet. 2004;74:160-7.

23. Tremlett H, Paty D, Devonshire V - Disability progression in multiple sclerosis is slower than previously reported. Neurology 2006. 24; 66:172-7.

24. Weinshenker BG, Rice GP, Nosworthy JH - The natural history of multiple sclerosis: a geographically based study. 3. Multivariate analysis of predictive factors and models of outcome. Brain. 1991; 114:104556.

25. Palace J, Duddy $M$, Lawton $M$ et al - Assessing the long-term effectiveness of interferon-beta and glatiramer acetate in multiple sclerosis: final 10-year results from the UK multiple sclerosis risksharing scheme. J Neurol Neurosurg Psychiatry. 2018; DOI: 10.1136/ jnnp-2018-318360.

26. Lublin FD, Baier M, Cutter G - Effect on relapses on developmental of residual deficit in multiple sclerosis. Neurology. 2003; 9:1528-1532.

27. Young PJ, Leaderer C, Eder Ket al - Relapses and subsequent worsening of disability in relapsing-remitting multiple sclerosis. Neurology 2006; 12 : 804-8.

28. Kappos L, Freedman MS, Polman CH, et al - Effect of early versus delayed interferon beta- $1 \mathrm{~b}$ treatment on disability after a first clinical event suggestive of multiple sclerosis: a 3-year follow-up analysis of the BENEFIT study. Lancet. 2007;370;389-97. 
29. Panitch H, Miller A, Paty D, Weinshenker B - Interferon beta-1b in secondary progressive MS: results from a 3-year controlled study. Neurology. 2004;63:1788-1795.

30. Cree BA, Gourraud PA, Oksenberg JR, et al - Long term evolution of multiple sclerosis disability in the treatment era. Ann Neurol. 2016;80:499-510

31. Vukusic S, Confavreux C - Prognostic factors for progression of disability in the secondary progressive phase of multiple sclerosis. J Neurol Sci. 2003;206:135-7.

32. Confavreux C, Vuksic S - Age at disability milestones in multiple sclerosis. Brain. 2006;129:595-605.

33. Trojano M, Pellegrini F, Fuiani A et al - New natural history of Interferon$\beta$-treated relapsing multiple sclerosis. Ann Neurol. 2007;61:300-6.

34. Bergamaschi R - Prognostic factors in multiple sclerosis. Int Rev Neurobiol. 2007;79:423-47.

35. Degenhardt A, Ramagopalan SV, Scalfari A, Ebers GC - Clinical prognostic factors in multiple sclerosis: a natural history review. Nat Rev Neurol. 2009; 5:672-82.

36. Visscher B, Liu KS, Clark VA, Detels R, Malmgren RM, Dudley JP -
Onset symptoms as predictors of mortality and disability in multiple sclerosis. Acta Neurol Scand. 1984;70:321-28.

37. Razzolini L, Portaccio E, Stromillo ML et al - The dilemma of benign multiple sclerosis: Can we predict the risk of losing the "benign status"? A 12-year follow-up study. Mult Scler Relat Disord. 2018; DOI: 10.1016/j. msaed.2018.08.011 Epub ahead of print.

38. Reader AT, Ebers GC, Traboulsee A, et al - Cross-sectional study assessing long-term safety of interferon-beta-1b for relapsing-remitting MS. Neurology. 2010;74:1877-85.

39. Zettl UK, Hecker M, Aktas O, Wagner T, Rommer PS - Interferon $\beta-1 \mathrm{a}$ and $\beta-1 \mathrm{~b}$ for patients with multiple sclerosis: updates to current knowledge. Expert Rev Clin Immunol. 2018;14:137-53.

40. Baum K, O'Leary C, Coret Ferrer F, et al - Comparison of injection site pain and injection site reactions in relapsing-remitting multiple sclerosis patients treated with interferon beta-1a or 1b. Mult Scler. 2007;13:115360.

41. Harris C, Billisberger K, Tillotson $L$ et al - Injection-site pain in patients with multiple sclerosis: interferon beta- $1 \mathrm{~b}$ versus interferon beta-1a. Int J MS Care. 2005/2006; 7:132-6. 